\title{
Interactive Visualization of 3D Redshift Surveys with SDvision
}

\author{
Daniel Pomarède and Marguerite Pierre \\ CEA/IRFU, Saclay \\ 91191 Gif-sur-Yvette, France \\ email: daniel.pomarede@cea.fr, marguerite.pierre@cea.fr
}

\begin{abstract}
The three-dimensional visualization of redshift surveys is a key player in the comprehension of the structuration of the cosmic web. The SDvision software package, intended primarily for the visualization of massive cosmological simulations, has been extended to provide an interactive visual representation of different classes of redshift surveys, with the objective to enable direct comparisons between the rare highest-density peaks traced by the clusters of galaxies found in the XMM-LSS Survey and the densely populated catalogues of galaxy photometric redshifts. We present the various possibilities offered by this tool in terms of filtering of the data, reconstruction of density fields, interactivity and visual rendering, including various techniques such as ray-casting, isosurfaces, slicing and texturing. This is illustrated using the C1 and C2 samples of the XMM-LSS Survey, and the publicly released COSMOS and CFHTLS photometric redshift Catalogs. Comparisons with published results are presented and discussed.
\end{abstract}

\section{Introduction}

Structures in the distribution of galaxies in the Universe are revealed by redshift surveys. These structures are found to form a cosmic web of clusters, filaments and walls delineating large voids (see e.g. Kovač et al. 2010 and references therein). This complex structuration is displayed using the benefits of three-dimensional, interactive visualization. The SDvision visualization software, initially developed to visualize numerical simulations of astrophysical plasmas, provides ways to explore the structures and compare the features of different classes of redshift surveys. The primary objective of this project consists in visualizing the clusters of galaxies identified in the context of the XMMLSS Survey (Pierre et al. 2004), with the intent to explore the existence of voids and large scale structures. In order to perform comparisons of these data with other surveys, this tool also provides access to the large-statistics photometric redshift surveys of the CFHTLS in the W1 Field. To provide some level of comparisons with published results, an interface to the COSMOS-2008 photometric redshift catalog is also available.

\section{The SDvision Visualization software}

The SDvision visualization interface (Pomarède et al. 2008) is developed has part of the COAST Computational Astrophysics Project which aims at high-performance simulations of astrophysical plamas (Audit et al. 2006). It is deployed in the framework of IDL Object Graphics, thus benefitting from both a wide collection of scientific functions and the hardware acceleration of its OpenGL interface layer. It can handle 3D scalar and vector fields defined on Cartesian grids, and clouds of points. Scalar fields are visualized by either ray-casting, isosurface reconstruction, slicing, or stacking of textures. Vector fields are visualized with streamlines or hedgehog displays. Point clouds are visualized as $3 \mathrm{D}$ scatter plots. An example of utilization of the widget is shown in Figure 1. 


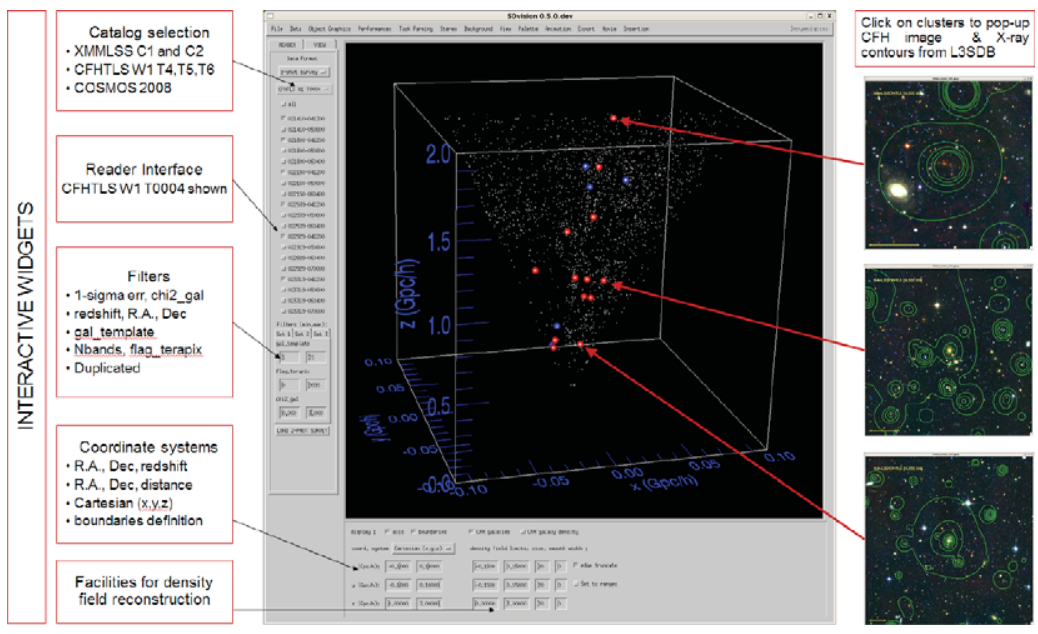

Figure 1. The SDvision graphical interface used here to visualize together samples of the XMM-LSS C1 and C2 galaxy clusters (blue and red spheres) and the CFHTLS photometric redshift survey of galaxies in the W1 field (white dots). Images on the right are popped up by clicking on the clusters, displaying together XMM X-ray contours and CFHTLS optical images.

\section{Visualization of the XMM-LSS Survey}

The XMM Large Scale Structures survey (XMM-LSS) is a medium deep large area X-ray survey which aims to address Large Scale Structures with galaxy clusters. This survey has provided two samples, the Class 1 and Class 2 cluster samples (Pacaud et al. 2007), for a total of $\sim 110$ clusters. These objects are visualized as Gouraud-shaded spheres as illustrated in Figure 1. This technique takes advantage of the hardware acceleration of the graphics card. The radius of the spheres can be set proportionally to the mass of the cluster. The interface offers several interactive filters to be applied to these samples : selection criteria ("confirmed", "provisional", "tentative", "photometric"), redshift, right ascension, declination. The view can be rotated, translated and zoomed at will to explore the dataset, including full immersion using wide angle projections. Output for stereoscopic display systems are available. To improve the insight given on these samples, images of the clusters are popped up by simply clicking the spheres. These images, showing the cluster X-ray contour and an optical observation, are obtained from the L3SDB database. The distribution of the $\mathrm{C} 1$ and $\mathrm{C} 2$ clusters is displayed in Figure 2 as an isometric view in the (R.A., Dec) projection.

\section{Visualization of photometric redshift surveys}

Two galaxy surveys are available for interactive visualization in SDvision :

CFHTLS in the W1 Field. The Canada France Hawaii Telescope Legacy Survey offers accurate and reliable photometric redshift catalogues (Coupon et al. 2009). The W1 wide field covers a region of $8^{\circ} \times 9^{\circ}$ that overlaps with the XMM-LSS field (Figure 2).

COSMOS-2008. The COSMOS Photometric Redshift Catalog Fall 2008 (mag 25 limited) provides 385065 redshift measurements in the 2-deg ${ }^{2}$ COSMOS field (Ilbert et al. 2009). Examples of visualization of these data are shown in Figure 3.

To visualize the $3 \mathrm{D}$ distribution of galaxies using the photometric redshifts, several ways are offered. The primary information that is the position of the galaxies is most simply viewed using a 3D scatter-plot (Figure 3). Filters can interactively be applied, such as the galaxy model used for the fit and the resulting $\chi^{2}$. A number density field can be 

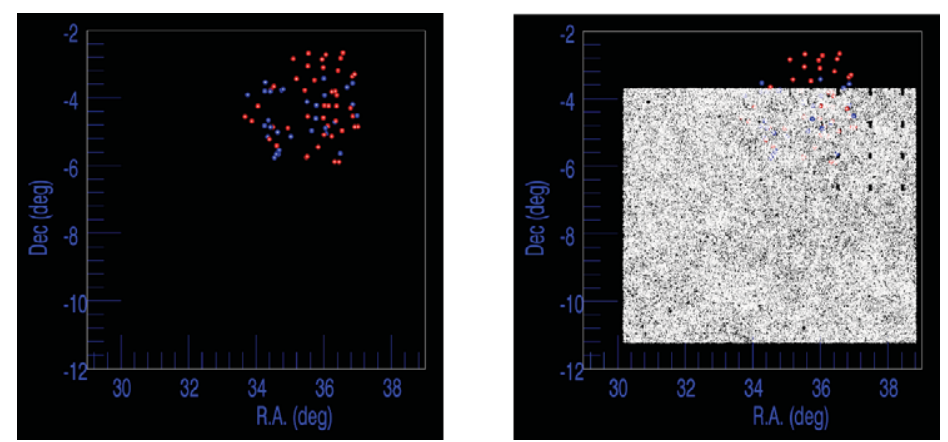

Figure 2. Left : visualization of the XMM-LSS clusters of C1 (red spheres) and C2 (blue spheres) samples. Right : the preceding view is added with the galaxies of the CFHTLS in the $\mathrm{W} 1$ field, with the filter gal_template $=1$.
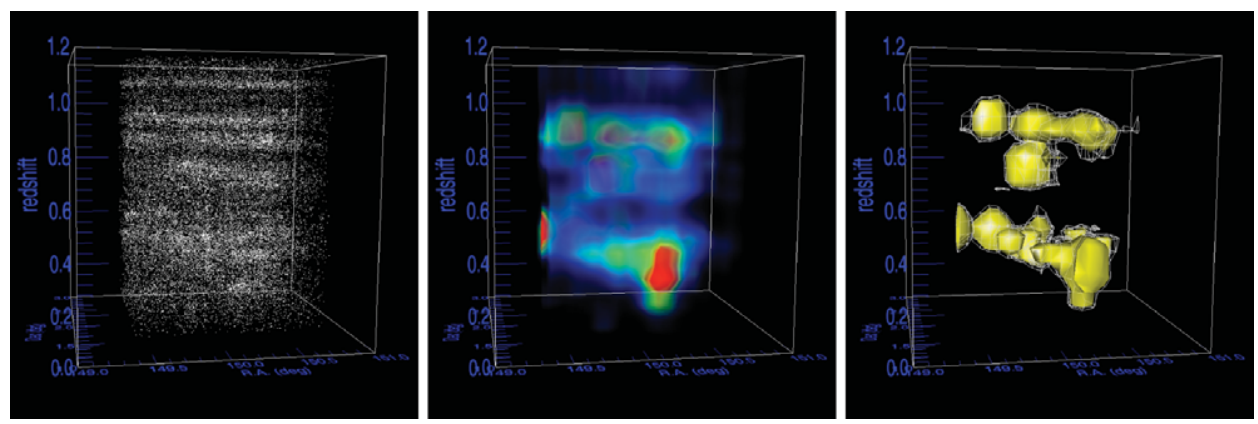

Figure 3. Visualization of the COSMOS-2008 redshift survey using different techniques, from left to right : 3D scatter-plot of the galaxies, reconstructed density field viewed by ray-casting, and by isosurfaces. The following filters are applied : $z \leqslant 1.2$, mod_gal $_{-} \leqslant 21$, chi_gal $\leqslant 2$.

reconstructed on a grid and smoothed using a simple boxcar average, where the box width can be set at will. The resulting field is visualized using the various techniques available in SDvision, as illustrated in Figure 3. The visualization of the COSMOS-2008 photometric redshift catalog is most useful to perform comparisons with published results. This is first illustrated in Figure 4 for the density distribution of LSS\#1 in Scoville et al. 2007, a large structure at $\mathrm{z}=0.73$ in a slice $\Delta z=0.27$ viewed here in the (R.A., Dec) projection. The high density peaks are observed in the same locations. We then compare our 3D density distribution with those presented using the spectroscopic redshifts of the 10k zCOSMOS galaxies in Kovač et al. 2010 and the LSS structures from Scoville et al. 2007, showing agreements on locations of the densest regions.

\section{Visual comparisons of XMM-LSS and CFHTLS}

The potential of SDvision in comparing visually the rare highest-density peaks traced by the clusters of galaxies found in the XMM-LSS Survey and the densely populated catalogues of galaxy photometric redshifts is illustrated in Figure 5. In this preliminary result, the $\mathrm{C} 1$ and $\mathrm{C} 2$ clusters of the confirmed class are displayed against a density field reconstructed using the T0006 release of the CFHTLS Catalog with the following filters : gal_template $\leqslant 21$, chi2_gal $\leqslant 10$., redshift $\leqslant 1.2,33.4 \leqslant R . A . \leqslant 37.4,-6 . \leqslant$ Dec $\leqslant-2$. The X-ray clusters are found to fall mostly in regions of high density of galaxies and are clearly associated with the "fingers of God" conspicuous in the galaxy distribution. 

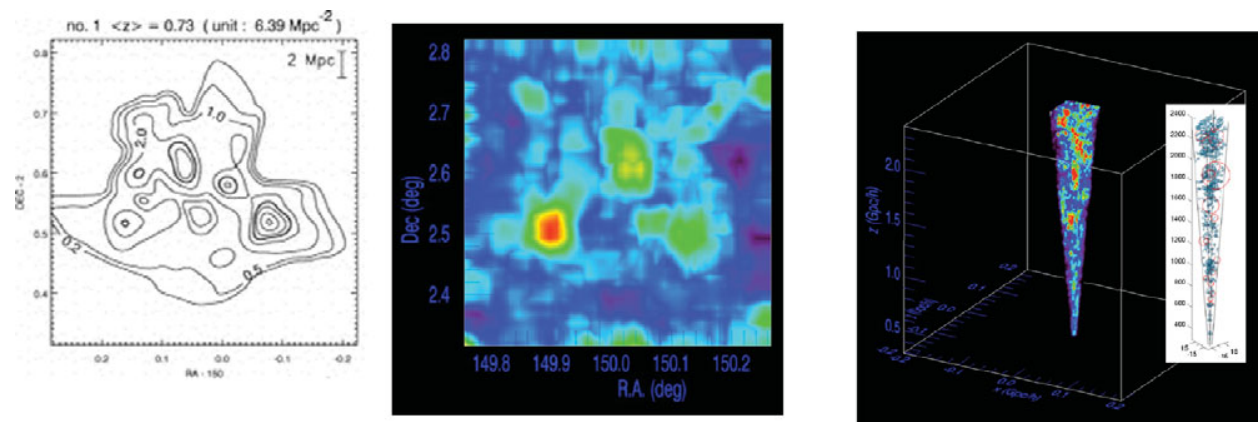

Figure 4. The two leftmost images compare the distributions obtained for the COSMOS photometric LSS\#1, while the rightmost plot provides a comparison with the 10k zCOSMOS density field and photometric structures (see explanations in text).

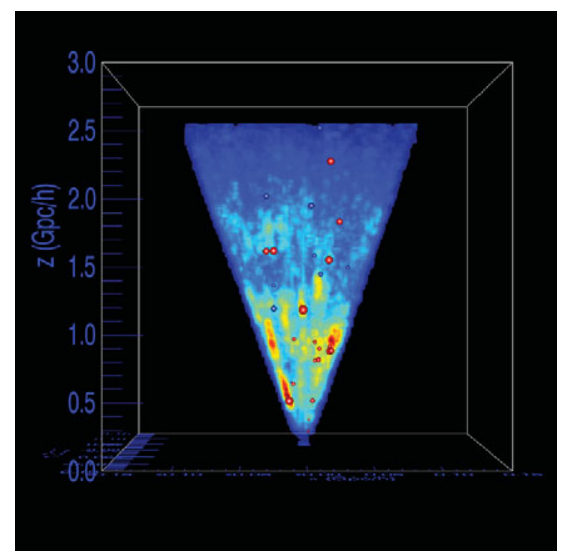

Figure 5. Visual comparison of the XMM-LSS and CFHTLS Surveys.

\section{Conclusion and perspectives}

A software tool aiming at the interactive and comparative visualization of redshift surveys of different classes has been developed. It is used to compare the XMM-LSS and CFHTLS current releases. It will be maintained against future releases of the catalogs. A major prospect is the visualization of the XXL Survey that will cover $50 \mathrm{deg}^{2}$ at a depth similar to the XMM-LSS (Pierre et al. 2011). A further application will be to investigate how the various classes of X-ray AGNs are located within the large scale structures.

\section{References}

Audit, E., Pomarède, D., Teyssier, R., \& Thooris, B., 2006, ASPCS, 359, 9

CFHTLS, http://www.cfht.hawaii.edu/Science/CFHLS/

Coupon, J., et al. 2009, A\&A, 500, 981

Ilbert, O., et al. 2009, ApJ, 690, 1236

Kovač, K., et al. 2010, ApJ, 708, 505

L3SDB, http://13sdb.in2p3.fr:8080

Pacaud, F., et al. 2007, MNRAS, 382, 1289

Pierre, M., et al. 2004, JCAP, 09, 011

Pierre, M., et al. 2011, MNRAS sub (arXiv:1009.3182)

Pomarède, D., et al. 2008, ASPCS, 386, 327

Scoville, N., et al. 2007, ApJS, 172, 150 